\title{
Extrinsic anomalous Hall conductivity of a topologically nontrivial conduction band
}

\author{
Hai-Zhou Lu and Shun-Qing Shen \\ Department of Physics and Centre of Theoretical and Computational Physics, The University of Hong Kong, \\ Pokfulam Road, Hong Kong, China
}

(Received 27 February 2013; published 28 August 2013)

\begin{abstract}
A key step towards dissipationless transport devices is the quantum anomalous Hall effect, which is characterized by an integer quantized Hall conductance in a ferromagnetic insulator with strong spin-orbit coupling. In this work, the anomalous Hall effect due to the impurity scattering, namely the extrinsic anomalous Hall effect, is studied when the Fermi energy crosses with the topologically nontrivial conduction band of a quantum anomalous Hall system. Two major extrinsic contributions, the side-jump and skew-scattering Hall conductivities, are calculated using the diagrammatic techniques in which both nonmagnetic and magnetic scattering are taken into account simultaneously. The side-jump Hall conductivity changes its sign at a critical sheet carrier density for the nontrivial phase, while it remains sign unchanged for the trivial phase. The critical sheet carrier densities estimated with realistic parameters lie in an experimentally accessible range. The results imply that a quantum anomalous Hall system could be identified in the good-metal regime.
\end{abstract}

PACS number(s): 73.43.-f, 75.50.Pp, 85.75.-d

Introduction. The anomalous Hall effect appears in ferromagnets as a transverse current induced by a longitudinal electric field. Different from the ordinary Hall effect, it is not driven by the Lorentz force acting on charge carriers in a magnetic field. Instead, it stems from the interplay of the spin-orbit coupling and time-reversal symmetry breaking. ${ }^{1}$ The anomalous Hall conductance has two distinct contributions, from the extrinsic and intrinsic mechanisms. The extrinsic mechanism originates from electrons near the Fermi surface when they are scattered by impurities. The intrinsic mechanism, on the contrary, is given by the Berry curvature of electrons below the Fermi surface, as a consequence of the spin-orbit coupling induced topological properties in Bloch bands. ${ }^{2}$ In particular, the intrinsic anomalous Hall conductance could be quantized in units of the conductance quantum when the Fermi surface lies in the gap between energy bands. Known as the quantum anomalous Hall effect, ${ }^{3,4}$ the phenomenon is a key step towards dissipationless quantum transport without magnetic field, and thus has attracted much effort for its experimental realization. ${ }^{5,6}$ One promising proposal is to magnetically dope a quantum spin Hall system, ${ }^{7,8}$ which can be regarded as two time-reversed copies of the quantum anomalous Hall system. The two copies have exactly opposite Hall conductances that cancel with each other. The magnetic doping, ${ }^{9-11}$ which breaks time-reversal symmetry, can lift the cancellation and give rise to the quantum anomalous Hall effect. However, the doping and inheriting defects always bring extra carriers, which shift the Fermi energy out of the gap and into an energy band where electron transport suffers from impurity scattering. In this situation, the extrinsic mechanisms also becomes relevant (see Fig. 1), but was never addressed.

In this work, we study the extrinsic anomalous Hall effect of the conduction band of a quantum anomalous Hall system. With the help of the Kubo formula and Feynman diagrams, we calculate the side-jump and skew-scattering contributions to the Hall conductivity, two major extrinsic mechanisms. We find that the side-jump Hall conductivity could change sign at an experimentally accessible sheet carrier density in the topologically nontrivial phase, while its sign remains unchanged in the trivial case. The skew-scattering
Hall conductivity show a similar behavior when nonmagnetic scattering dominates. The sign-changing feature may serve as a signature for the quantum anomalous Hall system in a dirty device.

Model. The minimal model for the quantum anomalous Hall system takes the form

$$
H=\gamma\left(k_{x} \sigma_{x}+k_{y} \sigma_{y}\right)+\left(\frac{\Delta}{2}-B k^{2}\right) \sigma_{z},
$$

where $\gamma=v \hbar, v$ is the effective velocity, $\left(k_{x}, k_{y}\right)$ is the wave vector, and $k^{2}=k_{x}^{2}+k_{y}^{2} . \sigma_{x / y}$ are Pauli matrices. $\Delta=m_{0} \pm$ $g M$, where $m_{0}$ is a gap opened at $k=0$ for the hybridized surface states of a three-dimensional topological insulator. ${ }^{12}$ $g M$ describes the magnetic-doping-induced exchange field, which may effectively modulate $\Delta$ between positive and negative values for a given $m_{0} \cdot^{3,4}$ The $B k^{2} \sigma_{z}$ term is a topological correction ${ }^{13,14}$ to the usual minimal model for the anomalous Hall effect. ${ }^{15-20} H$ has one conduction band $|+, \mathbf{k}\rangle$ and one valence band $|-, \mathbf{k}\rangle$, with the dispersion $\epsilon_{ \pm \mathbf{k}}=$ $\pm \sqrt{\left(\Delta / 2-B k^{2}\right)^{2}+\gamma^{2} k^{2}}$. We assume that the Fermi energy $E_{F}$ always crosses with the conduction band, where $E_{F}$ is measured from the Dirac point at $\epsilon_{ \pm \mathbf{k}}=0$.

Intrinsic Hall conductance. When the Fermi energy lies in the gap, the longitudinal conductance is zero; i.e., the system is insulating as the valence band is fully filled. However, there may exist an intrinsic quantum anomalous Hall conductance ${ }^{12,32}$

$$
\sigma_{x y}^{i(-)}=-\frac{e^{2}}{2 h}[\operatorname{sgn}(\Delta)+\operatorname{sgn}(B)],
$$

where $\operatorname{sgn}(x)=1$ if $x>0, \operatorname{sgn}(x)=-1$ if $x<0$, and $\operatorname{sgn}(x)=0$ if $x=0$. If $\Delta B>0$, the model is in the nontrivial phase as $\sigma_{x y}^{i(-)}$ has an integer anomalous Hall conductance in units of $e^{2} / h$. The integer, which corresponds to the chiral edge states in the gap, is the Chern number from the nontrivial topological properties of the valence band. The conduction band always has an opposite Chern number compared to the valence band and thus also carries the nontrivial topological properties. If $\Delta B<0$, the model is called topologically trivial with a zero anomalous Hall conductance. The importance of 


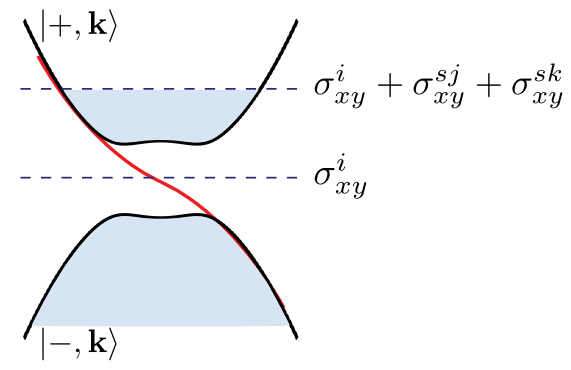

FIG. 1. (Color online) $\mid \pm$,k $\rangle$ represent conduction and valence bands of a quantum anomalous Hall system, respectively. When the Fermi energy (dashed) lies between the two bands, the anomalous Hall conductivity is governed dominantly by the intrinsic mechanism $\left(\sigma_{x y}^{i}\right)$, which is due to the chiral edge states (red solid). When the Fermi energy crosses with the conduction (or valence) band, the extrinsic mechanisms ( $\sigma_{x y}^{s j}$ for side jump and $\sigma_{x y}^{s k}$ for skew scattering) also contribute to the anomalous Hall conductivity.

$B k^{2} \sigma_{z}$ term deserves to be emphasized: With it, the solution of the in-gap chiral edge states can be found explicitly at an open edge. $^{22}$ Without the term, Eq. (2) gives a half-integer anomalous Hall conductance, ${ }^{15,16,21}$ and there is no edge-state solution at an open edge although the Jackiw-Rebbi bound state is allowed near a domain wall at which $\Delta$ changes sign. ${ }^{23}$

Side-jump Hall conductivity. Breaking time-reversal symmetry is indispensable for the anomalous Hall effect, so the anomalous Hall conductivity must depend on the time-reversal breaking terms in the model, such as $\left(\Delta / 2-B k^{2}\right) \sigma_{z}$ and magnetic scattering. The side-jump mechanism is related to the impurity scattering but not proportional to the total impurity concentration and scattering strength. ${ }^{1}$ As we will see, the side-jump extrinsic Hall conductivity is proportional to

$$
\cos \theta_{F}=\frac{\Delta / 2-B k_{F}^{2}}{E_{F}},
$$

where $k_{F}$ is the Fermi wave vector. This means that the side-jump Hall conductivity could change sign at a critical Fermi wave vector $k_{C}=\sqrt{\Delta / 2 B}$ if $\Delta B>0$, that is, if the system is in the nontrivial phase. In contrast, the Hall conductivity is monotonic if $\Delta B<0$, i.e., if the system is trivial. With the critical $k_{C}$, we can find the critical sheet carrier density $n_{C}=k_{C}^{2} /(4 \pi)$ and Fermi energy $E_{C}=v \hbar \sqrt{\Delta / 2 B}$. We estimate $k_{C}, n_{C}$, and $E_{C}$ with the experimental fitting data for topological insulator thin films and calculated parameters for HgTe quantum well, which are proposed host materials for the quantum anomalous Hall system. ${ }^{3,4}$ Table I shows the critical values of $k_{C}$ and $n_{C}$. In $10 \mathrm{~nm} n$-type $\mathrm{Bi}_{2} \mathrm{Se}_{3}$ thin films, ${ }^{27}$ only the surface states are populated for sheet carrier density below $5 \sim 7.7 \times 10^{12} / \mathrm{cm}^{2}$. Thus, most critical $n_{c}$ in Table I lie inside an experimentally accessible regime. The critical $n_{C}$ for HgTe is even much smaller. The above discussion shows again that the $B k^{2} \sigma_{z}$ term cannot be underestimated in a quantitative analysis. The first-principles calculations show that the bulk band minima will be pushed to higher energies in $\mathrm{Bi}_{2} \mathrm{Se}_{3}$ thin films ${ }^{4}$ (e.g., about $0.3 \mathrm{eV}$ for $5 \mathrm{QL}$ and $0.4 \mathrm{eV}$ for $3 \mathrm{QL}$ ), higher than corresponding $E_{C}$ in Table I. The higher order terms other than those in $H$ may shift the critical points, but will not qualitatively affect the sign-changing feature as long as they preserve time-reversal
TABLE I. Calculated critical Fermi wave vector $k_{C}$, sheet carrier density $n_{C}$, and Fermi energy $E_{C}$ with $\Delta, B$, and $v$ from experimental fitting data and $k \cdot p$ calculations. Entries $1-4$ are by Zhang et al. (Ref. 24), entry 5 is by Sakamoto et al. (Ref. 25), and entry 6 is by Konig et al. (Ref. 26). QL for quintuple layer is about $1 \mathrm{~nm} . \Delta$ in eV, $B$ in $\mathrm{eV} \AA^{2}, v$ in $10^{5} \mathrm{~m} / \mathrm{s}, k_{C}$ in $\AA^{-1}, n_{C}$ in $10^{12} / \mathrm{cm}^{2}$, and $E_{C}$ in $\mathrm{eV}$. The parameters in magnetically doped samples may be different. In magnetically doped cases, the exchange field could reduce $\Delta$, leading to smaller $n_{C}$.

\begin{tabular}{|c|c|c|c|c|c|c|}
\hline Sample & $\Delta$ & $B$ & $v$ & $k_{C}$ & $n_{C}$ & $E_{C}$ \\
\hline $2 \mathrm{QL} \mathrm{Bi}{ }_{2} \mathrm{Se}_{3}$ & 0.252 & 21.8 & 4.71 & 0.076 & 4.6 & 0.24 \\
\hline $3 \mathrm{QL} \mathrm{Bi}_{2} \mathrm{Se}_{3}$ & 0.138 & 18 & 4.81 & 0.062 & 3.1 & 0.20 \\
\hline $4 \mathrm{QL} \mathrm{Bi}_{2} \mathrm{Se}_{3}$ & 0.07 & 10 & 4.48 & 0.059 & 2.8 & 0.17 \\
\hline $5 \mathrm{QL} \mathrm{Bi}_{2} \mathrm{Se}_{3}$ & 0.041 & 5.0 & 4.53 & 0.064 & 3.3 & 0.19 \\
\hline $3 \mathrm{QL} \mathrm{Bi}_{2} \mathrm{Se}_{3}$ & 0.34 & 18 & 4.4 & 0.1 & 7.5 & 0.28 \\
\hline $7 \mathrm{~nm} \mathrm{HgTe}$ & -0.01 & -68.6 & 5.54 & 0.009 & 0.058 & 0.03 \\
\hline
\end{tabular}

symmetry. Also, the Berry phase is related to Eq. (3) by $\pi\left(1 \pm \cos \theta_{F}\right)$. It is known that the $\pi$ Berry phase leads to weak antilocalization in the longitudinal transport. ${ }^{28}$ The vanishing of $\cos \theta_{F}$ at the critical carrier density was predicted to give weak antilocalization if $\Delta B>0 .{ }^{29}$

The extrinsic Hall conductivity can be calculated by the Kubo formula in terms of the Feynman diagrams, where the impurity scattering is treated as the perturbation to the states $| \pm, \mathbf{k}\rangle$. The diagrams for the extrinsic anomalous Hall conductivity (see Fig. 2) have been systematically developed ${ }^{17}$
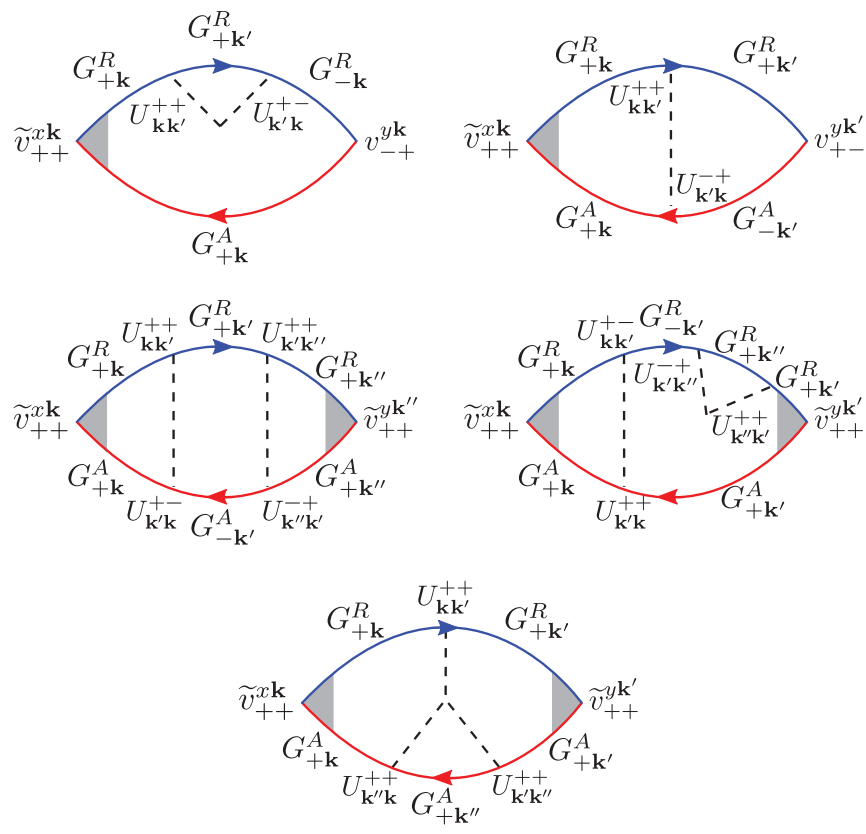

FIG. 2. (Color online) The diagrams for the extrinsic Hall conductivity can be summarized to only 5 of them. Top four: Side-jump contribution. Bottom: Skew-scattering contribution. \pm are band indices. $\mathbf{k}, \mathbf{k}^{\prime}, \mathbf{k}^{\prime \prime}$ are wave vectors. The left-bound and right-bound arrowed lines stand for retarded $\left(G^{R}\right)$ and advanced $\left(G^{A}\right)$ Green's functions, respectively. $U_{\mathbf{k}, \mathbf{k}^{\prime}}$ is the scattering matrix element. The dashed lines represent the correlation between scattering. $v^{x / y}$ are the bare velocities. The shadow area and tilde represent the vortex correction to the bare velocities. 
and applied to the model without the $B k^{2} \sigma_{z}$ term. ${ }^{17,18}$ Here we generalize the diagrammatic calculation by including the extra $B k^{2} \sigma$ term, and considering the nonmagnetic and magnetic impurities simultaneously. Despite lengthy calculation, we arrive at a compact form for the side-jump Hall conductivity ${ }^{32}$

$$
\sigma_{x y}^{s j}=-\frac{e^{2}}{h} \cos \theta_{F}\left[\frac{2 \alpha}{1-\alpha}+\frac{3 \alpha^{2} \eta_{B}}{2(1-\alpha)^{2}}\right],
$$

where $\cos \theta \equiv\left(\Delta / 2-B k_{F}^{2}\right) / E_{F}$,

$$
\alpha=\frac{\frac{1}{2}\left(1-V_{m} / V_{0}\right) \sin ^{2} \theta_{F}}{2-\sin ^{2} \theta_{F}+\left(V_{m} / V_{0}\right)\left(2+\sin ^{2} \theta_{F}\right)},
$$

and $\eta_{B}=1-2 B k_{F} /\left(\gamma \tan \theta_{F}\right) . V_{0} \equiv n_{0} u_{0}^{2}$ and $V_{m} \equiv n_{m} u_{m}^{2}$ here are of physical meanings. ${ }^{30,31} n_{0}$ and $n_{m}$ are the concentrations of nonmagnetic and magnetic impurities, respectively. $u_{0}$ and $u_{m}$ are spatially averaged strengths for the nonmagnetic and each component of the magnetic scattering, respectively. We have assumed isotropic magnetic scattering.

Figure 3 shows $\sigma_{x y}^{s j}$ in Eq. (4) for the quantum anomalous Hall and trivial cases. The horizontal axis is the sheet carrier density, which can be determined by the ordinary Hall measurement. As expected, $\sigma_{x y}^{s j}$ changes sign at the critical value of $n_{C}$ for the quantum anomalous Hall case [Fig. 3(a) $\Delta B>0$ ], while its sign remains unchanged for the trivial [Fig. 3(b) $\Delta B<0$ ] case. Besides, Fig. 3 also shows an impurity-related sign change in $\sigma_{x y}^{s j}$ as the ratio $V_{m} / V_{0}$ changes. $V_{0}$ and $V_{m}$ measure the nonmagnetic and each component of the magnetic scattering, respectively. Although the side-jump

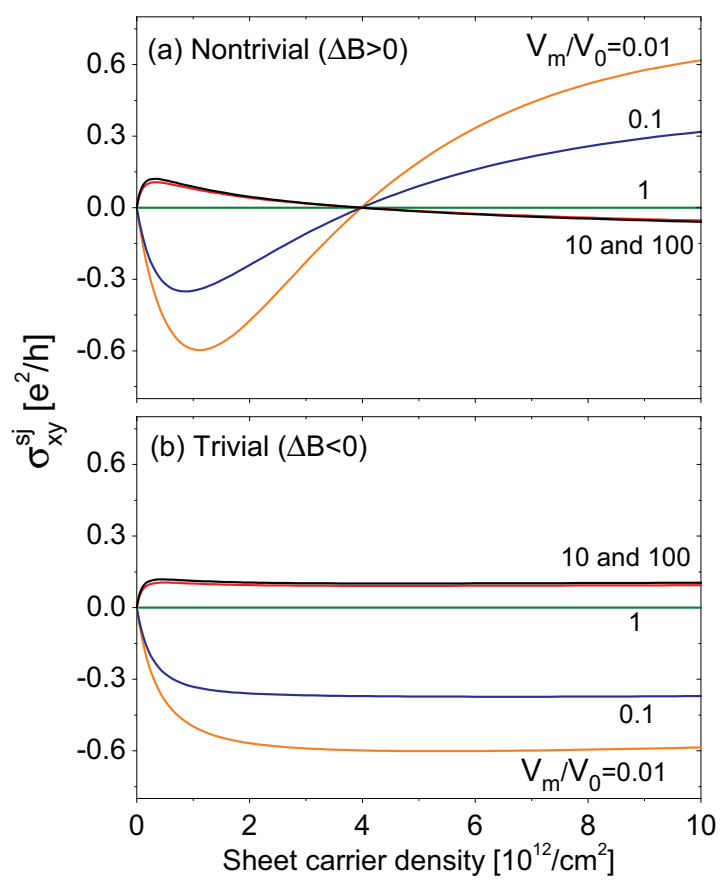

FIG. 3. (Color online) The side-jump Hall conductivity as a function of sheet carrier density. For all cases $\Delta=0.1 \mathrm{eV}$ and $v=4 \times 10^{5} \mathrm{~m} / \mathrm{s} . \quad B=10 \mathrm{eV} \AA^{2}$ for the nontrivial (quantum anomalous Hall) case (a) and $B=-10 \mathrm{eV} \AA^{2}$ for the trivial case. For better comparison, the parameters are not adopted directly from those in the experiments but of the same orders. $n_{C}$ is about $4 \times 10^{12} / \mathrm{cm}^{2}$ in this case.
Hall conductivity does not depends on the total scattering strength and impurity concentration, it may depend on the relative weight of different types of scattering. Varying $V_{m} / V_{0}$ gives rise to a sign change in $\sigma_{x y}^{s j}$ in both trivial and nontrivial cases in Fig. 3. This impurity-dependent sign change happens exactly at $V_{m}=V_{0}$, at which both $\alpha$ in Eq. (5) and $\sigma_{x y}^{s j}$ vanish.

Skew-scattering Hall conductivity. The skew-scattering Hall mechanism originates from the asymmetric scattering induced by the spin-orbit coupling. The leading order of the skew-scattering Hall conductivity can be calculated from the diagram in Fig. 2, and found as ${ }^{32}$

$$
\sigma_{x y}^{s k}=-\frac{e^{2}}{h} \frac{E_{F}\left(\eta_{B} \sin ^{2} \theta_{F}\right)^{2}\left(V_{3}^{0} \cos \theta_{F}-V_{3}^{z}\right)}{(1-\alpha)^{2}\left[V_{0}\left(2-\sin ^{2} \theta_{F}\right)+V_{m}\left(2+\sin ^{2} \theta_{F}\right)\right]^{2}},
$$

where the third-order impurity scattering correlations are defined as $V_{3}^{0} \equiv\left\langle U_{\mathbf{k k}^{\prime}}^{0} U_{\mathbf{k}^{\prime} \mathbf{k}^{\prime \prime}}^{0} U_{\mathbf{k}^{\prime \prime} \mathbf{k}}^{0}\right\rangle, V_{3}^{z} \equiv\left\langle U_{\mathbf{k} \mathbf{k}^{\prime}}^{z} U_{\mathbf{k}^{\prime} \mathbf{k}^{\prime \prime}}^{z} U_{\mathbf{k}^{\prime \prime} \mathbf{k}}^{z}\right\rangle$, with 0 and $z$ corresponding to the nonmagnetic elastic scattering and $z$ component of the magnetic scattering. $x$ and $y$ components are abandoned in the presence of the in-plane rotational symmetry. The above equation shows that only the nonmagnetic scattering part with $V_{3}^{0}$ is proportional to $\cos \theta_{F}$, so the skew-scattering shows the similar sign-changing feature only in absence of the magnetic scattering. $V_{3}^{0 / z}$ correspond to the correlation of three scattering events by one single impurity, so $V_{3}^{0 / z}$ are linearly proportional to the impurity concentration $n_{0 / m}$. Meanwhile the second-order scattering $V_{0 / m}$ are also linearly proportional to $n_{0 / m}$, so roughly speaking $\sigma_{x y}^{s k}$ is inversely proportional to the impurity concentration. For this reason, the skew-scattering Hall conductivity $\sigma_{x y}^{s k}$ is suppressed in a dirty metal.

Experimental implication. Because only the side-jump Hall conductivity always shows the sign change in the nontrivial phase, it is necessary to extract it among the three major contributions to the anomalous Hall conductivity. In principle, the intrinsic, side-jump, and skew-scattering

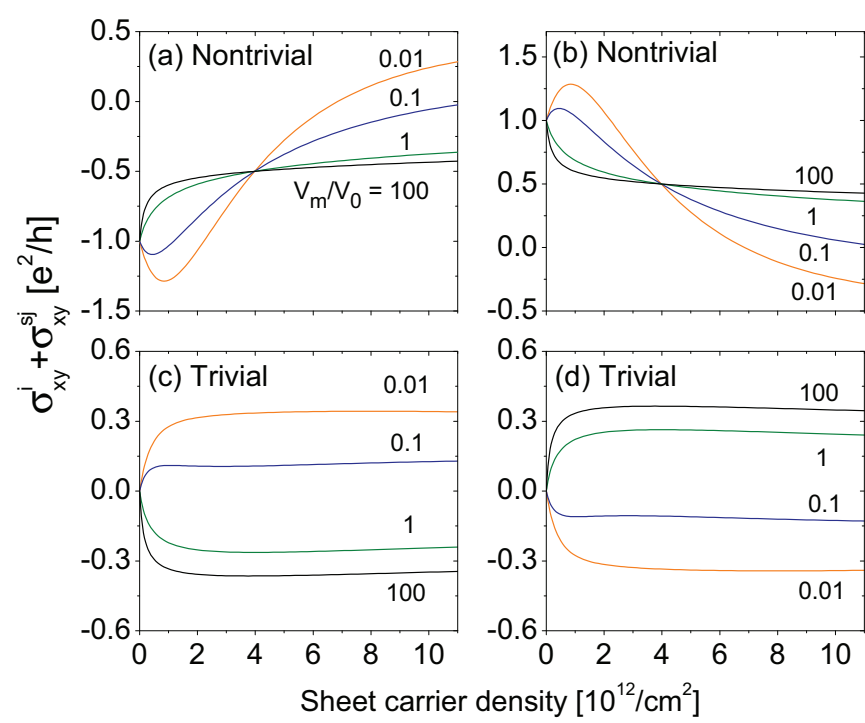

FIG. 4. (Color online) The sum of intrinsic and side-jump Hall conductivities as functions of sheet carrier density for different $V_{m} / V_{0}$. A square device is assumed so conductivity is equivalent to conductance. Parameters: $v_{F}=4 \times 10^{5} \mathrm{~m} / \mathrm{s}$, (a) $\Delta=0.1 \mathrm{eV}$, $B=10 \mathrm{eV} \AA^{2}$; (b) $\Delta=-0.1 \mathrm{eV}, B=-10 \mathrm{eV} \AA^{2}$; (c) $\Delta=-0.1$ $\mathrm{eV}, B=10 \mathrm{eV} \AA^{2}$; (d) $\Delta=0.1 \mathrm{eV}, B=-10 \mathrm{eV} \AA^{2}$. 
mechanisms can be distinguished in experiments. ${ }^{1}$ Empirically, the skew-scattering mechanism dominates in the high-conductivity regime where the longitudinal conductivity $\sigma_{x x}>10^{6}(\Omega \mathrm{cm})^{-1}$ and the anomalous Hall resistivity is proportional to the longitudinal resistivity. ${ }^{1}$ Considering the low conductivity in all the samples of $\mathrm{Bi}_{2} \mathrm{Se}_{3}$ family, the skew-scattering mechanism looks quite irrelevant for the recent experiments. ${ }^{5}$ In the good-metal regime where the Hall conductivity is independent of the longitudinal conductivity and $10^{4}<\sigma_{x x}<10^{6}(\Omega \mathrm{cm})^{-1}$, both the intrinsic and sidejump mechanisms could contribute. When the Fermi surface intersects the conduction band, the intrinsic mechanism also contribute a Hall conductivity ${ }^{12,32}$

$$
\sigma_{x y}^{i}=\sigma_{x y}^{i(-)}+\left.\sigma_{x y}^{i(+)}\right|_{E_{F}}=-\frac{e^{2}}{2 h}\left[\operatorname{sgn}(B)+\cos \theta_{F}\right] .
$$

Figure 4 shows the sum of $\sigma_{x y}^{i}$ and $\sigma_{x y}^{s j}$ as functions of the sheet carrier density of the conduction band. For the nontrivial phase, the curves of different $V_{m} / V_{0}$ cross at the critical sheet carrier density where $\sigma_{x y}^{s j}$ changes sign and the value of the Hall conductivity is shifted to $\left.\sigma_{x y}^{i}\right|_{n_{C}}+\left.\sigma_{x y}^{s j}\right|_{n_{C}}=-\frac{e^{2}}{2 h} \operatorname{sgn}(B)$. This shifting can be canceled if the time-reversed partner of $H$ is also considered, which gives $\operatorname{sgn}(-B)$. In contrast, those for the trivial case do not cross [Figs. 4(c) and 4(d)]. This crossing could provide an extra signature of the nontrivial phase, if the relative strength of the nonmagnetic and magnetic doping fluctuates accidentally or can be tuned in a controlled way from sample to sample. Also, the intrinsic and sidejump contributions can be separated by defining the intrinsic contribution as the extrapolation of the ac Hall conductivity to zero frequency in the limit of $\tau_{t r} \rightarrow \infty$, with $1 / \tau_{t r} \rightarrow 0$ faster than $\omega \rightarrow 0^{1} . \tau_{t r}$ is the transport time, which can be extracted from the longitudinal conductivity.

Summary. We show that extrinsic anomalous Hall conductivity in a topologically nontrivial conduction band (i.e., in the quantum anomalous Hall phase) exhibits different behaviors from those in a trivial band. More specifically, the side-jump extrinsic Hall conductivity changes sign at a critical sheet carrier density only in the nontrivial phase. When varying the ratio between nonmagnetic and magnetic scattering, the side-jump Hall conductivities cross at the critical sheet carrier density. The skew-scattering Hall conductivity shows similar behaviors when the nonmagnetic scattering overwhelms the magnetic scattering. These features may help future experiments that explore the quantum anomalous Hall systems.

Acknowledgment. This work was supported by the Research Grant Council of Hong Kong under Grant No. HKU705111P.
${ }^{1}$ N. Nagaosa, J. Sinova, S. Onoda, A. H. MacDonald, and N. P. Ong, Rev. Mod. Phys. 82, 1539 (2010).

${ }^{2}$ D. Xiao, M. C. Chang, and Q. Niu, Rev. Mod. Phys. 82, 1959 (2010).

${ }^{3}$ C. X. Liu, X. L. Qi, X. Dai, Z. Fang, and S. C. Zhang, Phys. Rev. Lett. 101, 146802 (2008).

${ }^{4}$ R. Yu, W. Zhang, H. J. Zhang, S. C. Zhang, X. Dai, and Z. Fang, Science 329, 61 (2010).

${ }^{5}$ C. Z. Chang, J. Zhang, M. Liu, Z. Zhang, X. Feng, K. Li, L. L. Wang, X. Chen, X. Dai, Z. Fang, X. L. Qi, S. C. Zhang, Y. Wang, K. He, X. C. Ma, and Q. K. Xue, Adv. Mater. 25, 1065 (2013).

${ }^{6}$ J. G. Checkelsky, J. Ye, Y. Onose, Y. Iwasa, and Y. Tokura, Nat. Phys. 8, 729 (2012)

${ }^{7}$ C. L. Kane and E. J. Mele, Phys. Rev. Lett. 95, 146802 (2005).

${ }^{8}$ B. A. Bernevig, T. L. Hughes, and S. C. Zhang, Science 314, 1757 (2006).

${ }^{9}$ Y. S. Hor, P. Roushan, H. Beidenkopf, J. Seo, D. Qu, J. G. Checkelsky, L. A. Wray, D. Hsieh, Y. Xia, S.-Y. Xu, D. Qian, M. Z. Hasan, N. P. Ong, A. Yazdani, and R. J. Cava, Phys. Rev. B 81, 195203 (2010).

${ }^{10}$ Y. L. Chen, J.-H. Chu, J. G. Analytis, Z. K. Liu, K. Igarashi, H.-H. Kuo, X. L. Qi, S. K. Mo, R. G. Moore, D. H. Lu, M. Hashimoto, T. Sasagawa, S. C. Zhang, I. R. Fisher, Z. Hussain, and Z. X. Shen, Science 329, 659 (2010).

${ }^{11}$ L. A. Wray, S. Y. Xu, Y. Xia, D. Hsieh, A. V. Fedorov, Y. S. Hor, R. J. Cava, A. Bansil, H. Lin, and M. Z. Hasan, Nat. Phys. 7, 32 (2011).

${ }^{12}$ H. Z. Lu, W. Y. Shan, W. Yao, Q. Niu, and S. Q. Shen, Phys. Rev. B 81, 115407 (2010).

${ }^{13}$ S. Q. Shen, W. Y. Shan, and H. Z. Lu, SPIN 1, 33 (2011).

${ }^{14}$ S. Q. Shen, Topological Insulators (Springer-Verlag, Berlin, 2012).
${ }^{15}$ S. Onoda, N. Sugimoto, and N. Nagaosa, Phys. Rev. Lett. 97, 126602 (2006).

${ }^{16}$ R. L. Chu, J. R. Shi, and S. Q. Shen, Phys. Rev. B 84, 085312 (2011).

${ }^{17}$ N. A. Sinitsyn, A. H. MacDonald, T. Jungwirth, V. K. Dugaev, and J. Sinova, Phys. Rev. B 75, 045315 (2007).

${ }^{18}$ S. A. Yang, H. Pan, Y. Yao, and Q. Niu, Phys. Rev. B 83, 125122 (2011).

${ }^{19}$ D. Culcer and S. Das Sarma, Phys. Rev. B 83, 245441 (2011).

${ }^{20}$ K. Nomura and N. Nagaosa, Phys. Rev. Lett. 106, 166802 (2011).

${ }^{21}$ X. L. Qi, Y. S. Wu, and S. C. Zhang, Phys. Rev. B 74, 085308 (2006).

${ }^{22}$ B. Zhou, H. Z. Lu, R. L. Chu, S. Q. Shen, and Q. Niu, Phys. Rev. Lett. 101, 246807 (2008).

${ }^{23}$ R. Jackiw and C. Rebbi, Phys. Rev. D 13, 3398 (1976).

${ }^{24}$ Y. Zhang, K. He, C. Z. Chang, C. L. Song, L. L. Wang, X. Chen, J. F. Jia, Z. Fang, X. Dai, W. Y. Shan, S. Q. Shen, Q. Niu, X. L. Qi, S. C. Zhang, X. C. Ma, and Q. K. Xue, Nat. Phys. 6, 584 (2010).

${ }^{25}$ Y. Sakamoto, T. Hirahara, H. Miyazaki, S. I. Kimura, and S. Hasegawa, Phys. Rev. B 81, 165432 (2010).

${ }^{26}$ M. König, H. Buhmann, L. W. Molenkamp, T. Hughes, C. X. Liu, X. L. Qi, and S. C. Zhang, J. Phys. Soc. Jpn. 77, 031007 (2008).

${ }^{27}$ D. Kim, S. Cho, N. P. Butch, P. Syers, K. Kirshenbaum, S. Adam, J. Paglione, and M. S. Fuhrer, Nat. Phys. 8, 459 (2012).

${ }^{28}$ H. Suzuura and T. Ando, Phys. Rev. Lett. 89, 266603 (2002).

${ }^{29}$ H. Z. Lu and S. Q. Shen, Phys. Rev. B 84, 125138 (2011).

${ }^{30}$ H. Z. Lu, J. Shi, and S. Q. Shen, Phys. Rev. Lett. 107, 076801 (2011).

${ }^{31}$ W. Y. Shan, H. Z. Lu, and S. Q. Shen, Phys. Rev. B 86, 125303 (2012).

${ }^{32}$ See Supplemental Material at http://link.aps.org/supplemental/ 10.1103/PhysRevB.88.081304 for the calculation details. 